\title{
Back to the future: team-centred, resource- based learning as the antecedent of computer-based learning
}

\author{
James E. Cresswell \\ Department of Biological Sciences, University of Exeter
}

\begin{abstract}
In this paper, I argue that gains can be made in both staff efficiency and educational value by replacing the traditional lecturelpractical format of higher-education courses with a new format involving teamcentred, resource-based learning ( $R B L)$. Under the new format, students are deployed in independent teams to tackle a series of tasks supported by various resources, including documentation, email access to a tutor and, of course, the team itself. The new format also fosters personal transferable skills (teamwork, time management), which are not directly addressed by the traditional lecturelpractical format. I further argue that whereas computer-based learning applications typically have a minor role as an adjunct to the traditional lecture format, they provide a natural medium for the delivery of resources in the RBL format. I illustrate my contention by reference to Bio. 1001, a course in fundamental science skills for first-year undergraduates. The results of student evaluations exemplify the success of the RBL format in Bio. 1001. Moreover, the new course returned substantial increases in staff efficiency. I describe the potential for computers to play a greater part in the course in future In conclusion, I speculate that the team-based, RBL format can be usefully generalized to any course that can be converted to continuous assessment by serial, group tasks.
\end{abstract}

\section{Introduction}

With respect to staff efficiency, it is hard to beat the traditional lecture as a format for a university course. With a folder of lecture notes, multiple-choice assessment and an optical mark reader, it is equally easy for the academic to process 10 or 100 or 500 students. Indeed, the example of many American universities suggests that class size need be limited only by the capacity of the auditorium.

The endurance of the lecture course is no accident. Computer-based learning (CBL) technology can claim to introduce 'added value' to the student experience as an adjunct to an existing lecture course, or to be well suited to students with special needs, such as distance learners, but the acceptance of CBL by those at the sharp end of teaching will be slow unless CBL can contribute to increased staff efficiency.

The traditional lecture course does, however, have an Achilles' heel: it fosters in students few of the Personal Transferable Skills (PTSs) that are now widely recommended to 
universities, e.g. teamwork, independent judgement, organizational skills (Dearing, 1997). Moreover, traditional lecture delivery can sometimes favour certain kinds of learning (comprehension, information recall) over others (synthesis, evaluation). Consequently, there is room to make improvements in educational efficiency if the cost in staff efficiency is not prohibitive. Below, I will describe my experience in replacing a traditional lecture format with one where independent student teams undertake resource-based learning (RBL), which means that the course content was delivered through media other than the lecturer. The idea behind RBL is not new (Noble, 1980; Clarke, 1982; Exley and Gibbs, 1994), but I will argue that it makes a natural antecedent of CBL because there is an obvious role for the computers to play: that of delivering the learning resources. Most importantly, the RBL format can actually increase staff efficiency by encouraging students to teach each other.

\section{Overview}

Bio. 1001, Biological Investigations, is a mandatory course for first-year undergraduates in the Department of Biological Sciences at the University of Exeter. The course caters for around 120 students and is supported by six postgraduate demonstrators (15 hours each) and one lecturer ( 35 contact hours). The IT facility is available in business hours only, and offers 12 PCs with typical core software. In previous incarnations, the course took the traditional lecture/practical format with two lectures per week over 10 weeks and five practical sessions of three hours each. The lectures were generally unsuccessful because of the heterogeneity in student backgrounds. Experienced students appeared to be bored by remedial teaching, and inexperienced students baffled by anything too advanced. Accommodating all students in a single practical session was also unsatisfactory because of the requirement that students share a computer terminal, which did not encourage everyone to obtain hands-on experience, and some students became marginalized and unmotivated. The revised course retained its 10 -week timetable position and credit value, but involved a redirection of the content away from pure statistical analysis, and a radical change in course format. The aim of the revised course was for students to be able to conduct a scientific experiment, to analyse the results, and to write a report on their experiment as a 'publication-quality' scientific paper. In this process, the students should acquire competence in the appropriate computer-based professional tools: spreadsheet, statistical package and word processor.

\section{Learning environment}

The key to the new course is in its organization. Approximately 120 students are grouped into 18 teams of 6 or 7, which are formed from the pastoral tutorial groups that the students join when they arrive in the department. Each team is assigned a postgraduate demonstrator to act as a mentor. The mentor will advise and assess the team. Each demonstrator acts as mentor to three teams. The cohesion of the course is maintained by email. Students can contact each other, their mentor or the lecturer, although all transactions between teams and the staff are mediated initially by mentors. Thus, mentors act as a filter between the students and the lecturer, to whom mentors report any problems either by email, or at a weekly meeting convened by the lecturer.

There are several advantages to deploying students in teams: 
- Resources can be distributed to teams instead of to individuals, which means that the resource budget is divided into 18 rather than 120 units. Consequently, a higher quality of documentation can be afforded.

- Students are encouraged to develop team-related PTSs.

- The heterogeneity in student backgrounds can be put to work when the experienced students help the inexperienced students in their team.

An important ingredient is that the students are assessed as a team for a major portion of the course marks. Therefore, individuals should be concerned for their team's performance, and this could motivate them to share their skills.

\section{Course work and its assessment}

The teams are set a series of 10 tasks, which involve either practice on software or, eventually, analysis of their own experimental results and the preparation of the scientific paper. The tasks are designed to build skills cumulatively towards the production of the scientific paper. Therefore, students learn email first, then progress through exercises that use the spreadsheet, the statistics package and, finally, the word processor. Each task is completed by each individual, but students can double their marks if the entire group completes the task by the deadline (typically the end of the week on which the task was issued). In this way, students are encouraged to help other group members. Often, the completion of a task will involve sending output from an application (for example, the spreadsheet) to the mentor by email. By email, the student receives prompt acknowledgement and, when necessary, advice on how to repeat the task satisfactorily.

Some of the tasks involve a group assessment, which proceeds as follows:

The entire group convenes at a time agreed with the mentor. Only if the entire group is present does the mentor then 'randomly' select two or three students for assessment, in which they are observed in performing some simple computer-based tasks. Again, all students must perform satisfactorily for the group to pass the assessment. The observational assessments help to ensure that all students have acquired competence, and reduce the possibility that some students are completing tasks for others. A final component of the assessment is the submission of the scientific paper itself, which is co-authored by each team except for the discussion section. Each student writes and submits his or heir own discussion, something which allows space for individuality. Thus, each student receives a mixture of team marks and individual marks for the scientific paper.

\section{Resources available to students}

In addition to providing a brief, the task sheets contain an appendix with hints and references to the course pack. In this instance, the course pack forms the heart of the RBL approach. Each learning group receives a course pack, which contains rules and resources. Rules specify the protocol for the assessments and the usage of resources (for example, a process for booking computer time, or access to mentors), and the rules for taking a group assessment. Resources in the course pack include various instructions for computer applications, exercises in the use of statistical tests, and guidance in conducting experiments and writing reports. Course pack documentation included material on 
software usage from IT Services at Exeter and also copies of various Student's Guides (from Software Made Simple, Leicester).

Students are also encouraged to attend a weekly organizational meeting at the lecture theatre, where there are facilities for displaying computer output to the audience. Here, they can watch a demonstration of the steps required to complete each task and they have a chance to ask questions in person. The most important resource is the student's own team, which acts as a learning group. In their team, students can pass on their own previous experience or collaborate in solving course-related problems. Students can also question their mentor by email, or book an advisory meeting, although the teams are made aware of time limits (not more than 30 minutes mentor time per team per week). If necessary, students can contact the lecturer by email with the offer of an appointment if necessary.

\section{Student performance in 1997}

Overall, the students' performance was very good, the mean mark for the course being 66 per cent, which was much higher than in preceding years. Three quarters of students completed all 10 tasks and 96 per cent completed at least seven. The continuous assessment allowed early identification of wayward students, and their tutor was immediately notified. This procedure proved highly effective in re-directing under-performing students. Without exception, the few team conflicts were resolved amicably by the rapid intervention of the pastoral tutor or lecturer (students were very quick to alert their mentor to difficulties that lost them points, which usually resulted from an inability to convene the entire group for observational assessments).

The students' perception of their own competence in scientific skills (experimental design, statistics, reports) was improved significantly by their experience of the course (Table 1), which is an important result because these are areas that, for undergraduates, are often surrounded by anxiety. With respect to IT skills, the students' own perception of their abilities rose greatly across the course and, by the end, they showed widespread confidence (Table 1). It is reasonable to assert that the course had provided satisfactory IT training for the entire cohort (with the exception of WWW-related activities).

\begin{tabular}{|lcl|}
\hline Skill area & Before & After \\
Windows & 1.5 & 1.9 \\
Email & 1.1 & 1.9 \\
Word processor & 1.1 & 1.8 \\
Computers & 1.0 & 1.5 \\
Spreadsheets & 0.5 & 1.5 \\
Experimental design & 0.5 & 1.1 \\
Statistics & 0.5 & 1.0 \\
Reports & 0.5 & 1.1 \\
File transfer & 0.2 & 1.4 \\
Statistical package & 0.1 & 1.0 \\
\hline
\end{tabular}

Table 1: Results of self-assessment questionnaires completed by 62 students before and after participating in Bio. 100I. Students were asked to grade their competence in each skill area as either 0 (no familiarity), I (fair), or 2 (good). The numbers show the mean of the scores. 


\section{Staff efficiency and performance}

As lecturer, it took me about two weeks to design the course by conversion from the previous lecture format and to prepare the course materials. During the course, I met with students (one hour per week) and postgraduate mentors (also one hour per week). In addition, I spent some time in answering email, dealing directly with some groups and in notifying tutors of under-performing students. Overall, I expect the new format to reduce my time input for the entire course from 35 contact hours to $25-30$ hours. The postgraduate demonstrators were satisfied with the requirements made of them, and mostly preferred to work via email at their own convenience rather than having to attend a practical session for an entire afternoon. From my point of view, the performance of demonstrators was much more equitable, being less dictated by their inclination/ability to deal directly with students in a laboratory setting.

\section{Possibilities for the development of CBL in Bio. $100 \mathrm{I}$}

The course is now at a stage where further support could be provided for the teams in tackling the tasks set for them. Computers provide a natural medium for delivering the supporting resources. For example, the STEPS consortium offers a variety of learning materials for the computer that relate to statistics and that are set in a biological context (Mogey, 1996, 23). Additionally, some form of computer-mediated self-assessment would offer opportunities for students to put their learning into practice.

\section{Conclusions}

Team-centred RBL appears to be an effective avenue for changing from lecture-centred delivery. In addition to improving staff efficiency, it also fosters a wide range of PTSs in the students. Moreover, the direct involvement of RBL provides a role for computers that are a natural choice for the delivery of the required resources to independent learners.

It is possible to argue that the format of Bio. 1001 will not generalize because many of the tasks are, in themselves, computer-based, and because the assessment of the tasks is pass/fail. However, these objections do not relate to the fundamental requirements for the adoption of the format, which is actually contingent on the ability to set teams to work on a series of tasks with adequate supporting resources. Therefore, it may be possible to introduce serial, group tasks into any continuously assessed course in which the intended learning outcomes can be accurately and explicitly identified and then logically ordered. I thus contend that the potential advantages of team-centred $R B L$ are sufficient to make its consideration worthwhile.

\section{References}

Clarke, J. (1982), Resource-based Learning for Higher and Continuing Education, London: Croom Helm.

Dearing, R. et al (1997), Higher Education in the Learning Society, Report of the National Committee of Inquiry into Higher Education. London: NCIHE Publications (HMSO), July.

Exley, K. and Gibbs, G. (1994), Course Design for Resource Based Learning Oxford: Oxford Centre for Staff Development. 
ALIJ Volume 6 Number 1

Mogey, N. (1996), Learning Technology Dissemination Initiative - Information Directory, 9th edition, Edinburgh: Herriot Watt University.

Noble, P. (1980), Resource-based Learning in Post-compulsory Education, London: Kogan Page. 